\title{
On the experimental study of the flow around a fishing net
}

\author{
Géraldine Pichot $^{\mathrm{a},{ }^{*}}$, Grégory Germain ${ }^{\mathrm{b}}$ and Daniel Priour ${ }^{\mathrm{a}}$ \\ a IFREMER, Technopôle Brest Iroise, 29280 Plouzané, France \\ b IFREMER, Centre de Boulogne, 150, Quai Gambetta, BP 699, 62321 Boulogne-sur-Mer, France \\ *: Corresponding author : Pichot G., Tel.: +33 (0)2 232365 46; fax: +33 (0)2 232367 90, email \\ address : Geraldine.Pichot@ifremer.fr
}

\begin{abstract}
:
Selectivity of fishing gears like trawls is obviously conditioned by the fish behaviour but also by the net and the flow. As well, flow may affect fish behaviour. With the aim of a better understanding of the flow in presence of a net and a catch, we suggest, through the experimental study below, to determine and to analyze the flow over a rigid cod-end in two configurations: with a closed and an open net entrance. Trials took place in a free surface flume tank and the flow characteristics were determined from velocity measurements obtained by laser velocimetry (inside, outside and through the meshes of the stiff cod-end). The experimental device, the measures and their analyzes are given here.
\end{abstract}

Keywords: Fluid mechanics; Hydrodynamics; Experimental study; Fluid structure interactions; Laser velocimetry; Turbulence

\section{Introduction}

Generally speaking, during fishing operations, juveniles, small fish or non-marketable fish are accidentally caught. These non-target fish are dead when discarded over- board. This leads to a waist for future fishing operations and contributes to a decline of marine resources [4]. Selectivity is the ability of fishing gears to prevent such by- catches. Bottom and mid-trawls are known to have a pretty poor selectivity [2]. It is also kwown that trawl selectivity occurs mainly in the rear part, named the codend [3]. Many studies are carried out each year in order to improve cod-end selectivity, for example by testing different mesh sizes or different mesh numbers. These studies are based on sea trials which must be numerous to avoid the effect of non controllable 
parameters such as fish population entering the trawl. Consequently these studies are expensive and sometimes unconclusive. One cheap way to proceed is to perform numerical simulations to study some specific aspect of cod-end selectivity. Softwares that govern the net deformations already exist, like FEMNET [18] or the one developed by Le Dret et al. [13]. Also fish behaviour softwares exist like behavioRis [5] and PRESEMO [8], [9]. A promising flow model is given by Lewandowski and Pichot $[10],[11],[17]$. It comes with a turbulent model and a permeability parameter that has to be fitted.

The present paper describes the experimental studies undertaken over these last years. They were motivated by the need of a physical understanding of the flow around a cod-end net and the need of test cases for numerical developments [10], [11], [12]. This is of great interest for selectivity studies insofar as the escape process is often partly passive [8]. Then the flow is one determining factor in the success of fish escape attemps.

A specific stiff cod-end was developed to avoid instabilities during flume tank tests. This structure is perfectly stable in the flow and its geometrical shape is known. Notice that during a trawl, one has to take into account the effects of sea state induced vessel motion, that are shown to play a significant role on selectivity [14]. The structure we consider here has allowed to collect data on the flow by Laser Doppler Velocimetry (LDV) and Particle Image Velocimetry (PIV) techniques. Velocity profiles, as well as punctual estimations of the turbulent kinetic energy are given. Two situations are under consideration, the first one deals with an open net entrance, and the second one with a closed one, the cod-end net entrance being closed with a cap.

The choice of the second configuration needs some explanation. Here only the codend net part is under study. But, in reality, it is preceded by an extension piece which is a cylindrical-shaped piece of netting. The unknown is the amount of fluid entering the cod-end net. As we shall see in the following, this significantly modifies the inner net flow. Building an extension piece would have been costly. So we opted for another configuration and the decision was taken to close the net entrance with a cap. It will not lead to the same flow as that obtained with an extension piece but, at least, a second validation test case is available, quickly and in a cheap way. Moreover such experiments give information on the nature of the flow as well as on the influence of the fluid entering the cod-end net on the inner net flow. A forthcoming work would be to plan new experimental campaigns to quantify the amount of fluid entering the cod-end. Notice that some sea measurements already performed tend to predict a velocity inside the net above the cod-end close to the trawling velocity [19]. 


\section{Experimental device}

\subsection{Experimental setup}

The experimental study took place in the IFREMER (French Research Institute for Exploitation of Sea) free surface flume tank in Boulogne-sur-Mer, France. The flume tank (Fig. 1) is $18 \mathrm{~m}$ long by $4 \mathrm{~m}$ wide and $2 \mathrm{~m}$ deep with a side observation window of $8 \mathrm{~m} \times 2 \mathrm{~m}$. The flow turbulence rate is less than $5 \%$ and the flow velocity ranges from 0.06 to $2.1 \mathrm{~m} / \mathrm{s}$.

Fig. 1. Presentation of IFREMER facility

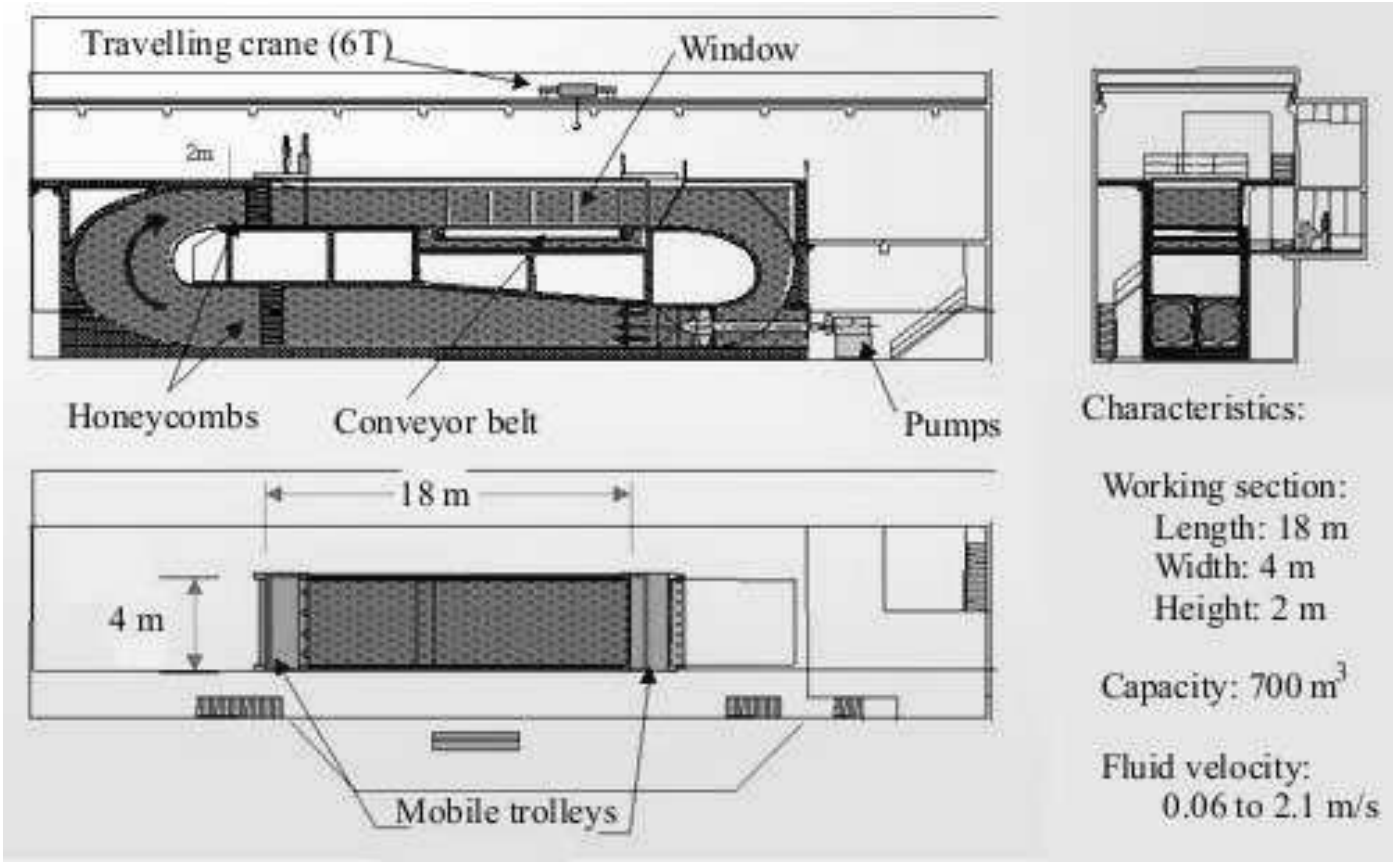

As the selectivity takes place mainly in the cod-end, we focus our work on this part of the trawl. The drag of a cod-end and the resulting twine tensions influence the overall cod-end geometry and the enlargement of the meshes [15]. In order to measure the shape and tension in a cod-end net, tests have been carried out for few netting characteristics, several catches and types of cod-ends. These preliminary tests are not described in the present paper. During these tests, the external shape of a $1 / 3$ scaled model of a 6 meter long cod-end has been measured. Unfortunately, the flow could not be measured during this first phase due to the instabilities of the cod-end and especially of the catch inside the structure. Thus, a specific stiff cod-end has been developed to avoid instabilities [7]. The structure is axi-symmetric and the catch inside the cod-end is limited by two sherical caps, so its shape is known. The $1 / 6$ scaled model is $1 \mathrm{~m}$ long, made of PA twine of $1200 \mathrm{~m} / \mathrm{kg}$, with diamond-shaped meshes of $30 \mathrm{~mm}$ mesh side and set at the bottom of the IFREMER flume tank. For this study, a medium-sized catch is considered in order to be representative of a typical configuration (or at least an intermediate step during fishing operations). The catch is $30 \mathrm{~kg}$, which gives an external diameter of $454 \mathrm{~mm}$. The stiff cod-end 
consists of 36 meshes on the perimeter and 21.5 meshes long. The water speed is $0.51 \mathrm{~m} / \mathrm{s}$. At real scale (at sea), this gives a $6 \mathrm{~m}$ long cod-end, a catch of $6480 \mathrm{~kg}$ and a towing speed around 2.5 knots.

\subsection{Instrumentation}

Two non-intrusive optical measurement devices are used to characterize the flow over the stiff cod-end:

- a two components Laser Doppler Velocimetry (LDV) for local measurements,

- a two components Particle Image Velocimetry (PIV) technique for global information on the flow.

The seeding particles used for the experiments are $15 \mu \mathrm{m}$ in diameter.

The LDV technique is a non-intrusive technique used to achieve velocity measurements at single points. It is based on the Doppler effect. Fig. 2 displays the LDV device. Let us denote $(O, x, y, z)$, the global frame of reference, its origin being set at the entrance of the net model. The available LDV device allows to measure two velocity components thanks to two wavelengths $(514.5 \mathrm{~nm}$ and $488 \mathrm{~nm})$. The measured components are the ones along the $x$ and $z$ directions.

Fig. 2. LDV system

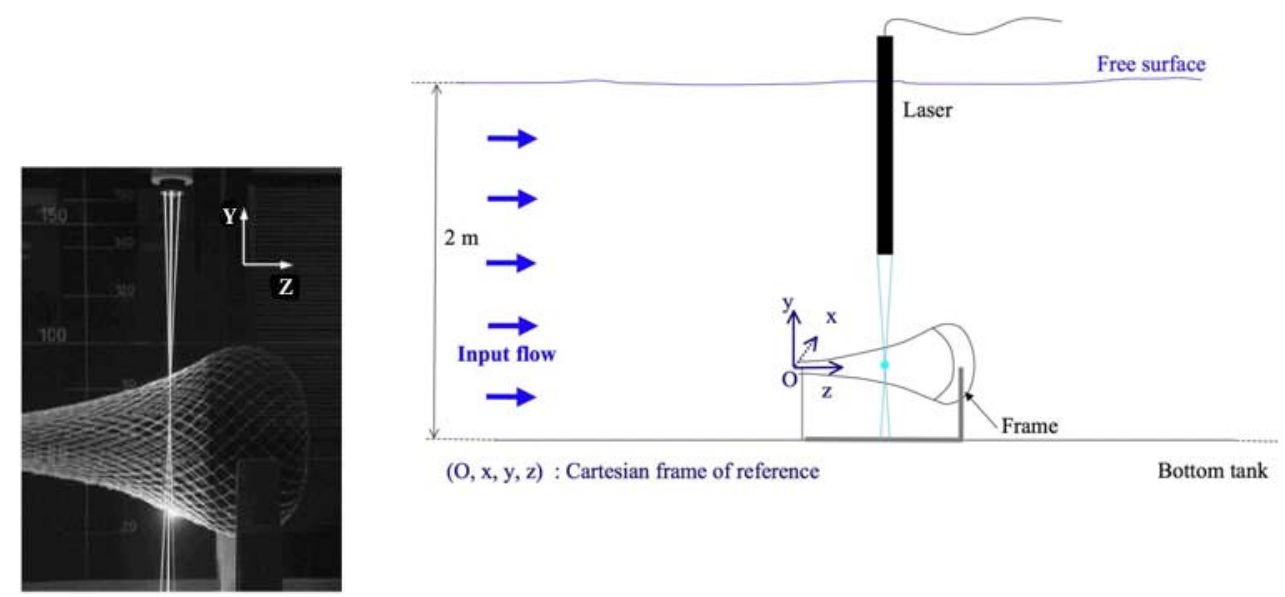

A particular feature of the LDV measurements is that the number of data recorded in a given time window is strongly dependent on the local seeding conditions [6]: measurements are possible only when a particle moves across the probe volume. Consequently, there are some regions (clean flow) where acquisitions with a relatively high data rate were possible (exceeding $50 \mathrm{~Hz}$ ); on the other hand, close to the walls or near recirculating zones the rate falls to very low values (lower than $10 \mathrm{~Hz}$ ). In order to achieve samples of data as homogeneous as possible, an inhibit method was used and data were recorded under time rather than sample length control. This technique allowed to obtain a sample length never exceeding 100 seconds (which is 
an order of magnitude for the time window larger than the time scale of the flow fluctuations) with a number of data per sample never exceeding 5000. The long time span allows an accurate estimate of average values for velocity and turbulence intensity.

The PIV technique provides information over a flow field, simultaneously at many points. It is based on illuminating the seeding particles and storing the resulting camera images to analyse displacements of particles between two successive images. The velocities are obtained by dividing the distance by the elapse time of laser pulses. The laser used is a two-chamber Gemini PIV Nid-Yag 2 x $120 \mathrm{~mJ}$ at $15 \mathrm{~Hz}$ pulsed laser. The camera is a Hi-sense, $1280 \times 1024$ pixels $^{2}$, with a focal lens length of $60 \mathrm{~mm}$ with a filter wavelength of $3 \mathrm{~nm}$. The measurement plane is typically 260 x $220 \mathrm{~mm}^{2}$. The image processing is done with the software Flow Map 1500 from Dantec dynamics. It is based on image intercorrelation on regions of $32 \times 32$ pixels $^{2}$, with a covering rate of $25 \%$. The vector field was evaluated by a predefined velocity magnitude and the invalid one were replaced by the moving average method. Instantaneous velocity fields were obtained and generally, a series of instantaneous measurements were statistically average to get the mean velocity field. The instantaneous and mean velocity fields and turbulence quantities are of great practical interests.

\subsection{Experimental protocol}

The aims of these experimental campaigns are multiple :

(1) Emphasizing the turbulent nature of the flow;

(2) Supplying LDV profiles in two different situations (open and closed net) yielding two different test configurations to validate numerical simulations;

(3) Understanding the interactions between the inner net flow and the external one.

So data were collected in two different situations. First, we completed measurements in an open net configuration. Then, the entrance of the net was closed by a cylindrical-shaped foam and measurements were performed by the LDV technique to estimate the impact on the internal flow (Fig. 3).

For both cases, mean velocity profiles as well as punctual turbulence intensity were collected using the non-intrusive techniques previously described. PIV images were stored in the case of the open net entrance to emphasize the turbulent feature of the flow. Velocity profiles were collected along the six profiles drawn on figure 4 [7]. Punctual turbulence intensity measures were gathered in the two configurations. Point positions are given in figure 4. 
Fig. 3. Net model with a closed entrance and its frame set at the bottom of the IFREMER flume tank

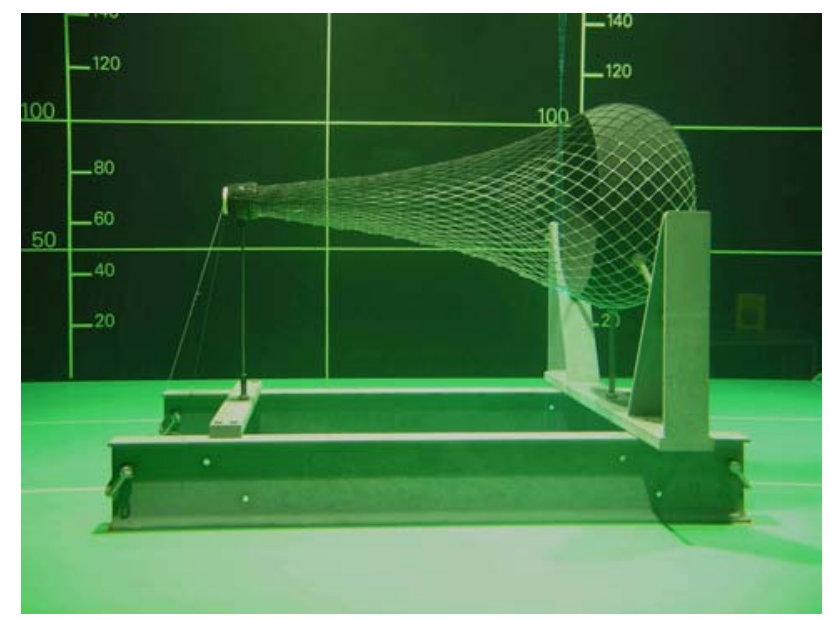

Fig. 4. Locations of the measurement profiles and dot positions for punctual LDV measurements

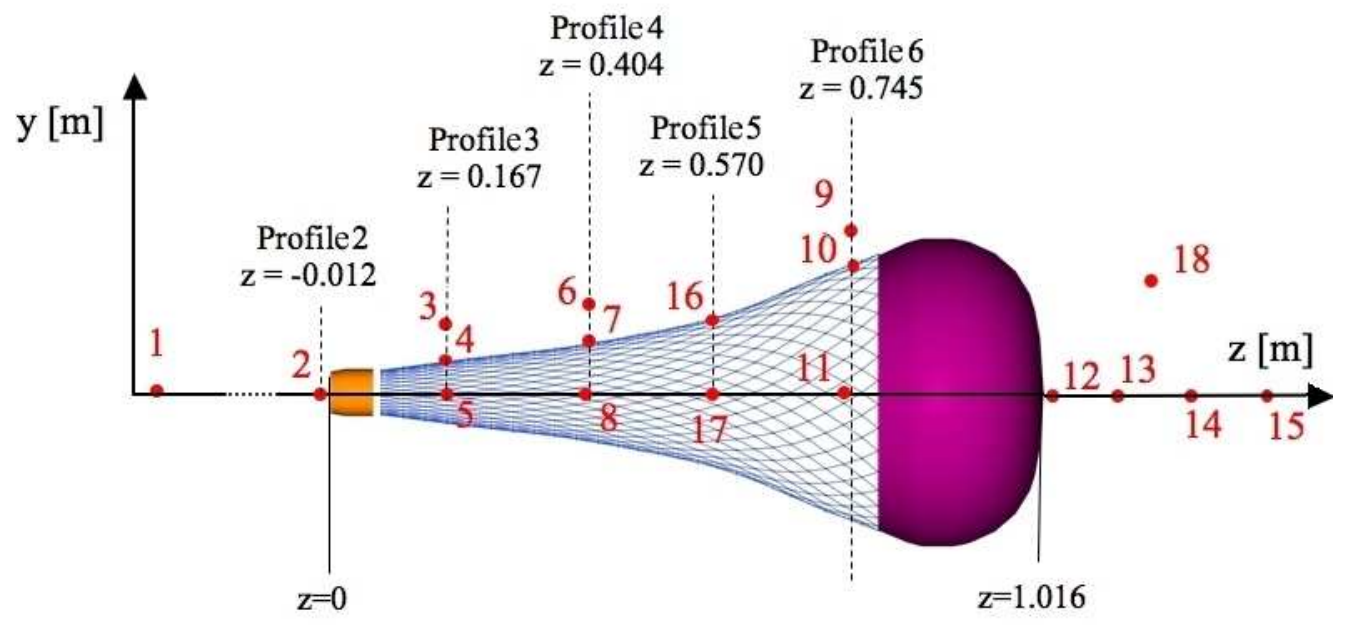

\section{Open net model}

\subsection{PIV results}

An indicator of the turbulence feature of the flow is the Reynolds number. In the vinicity of the catch, it can be computed using the maximal diameter of the catch $(0.45 \mathrm{~m})$ as the reference length $L$ and the input velocity $(0.51 \mathrm{~m} / \mathrm{s})$ as the reference velocity $U$ :

$$
R e=\frac{U L}{\nu}=2.10^{5}
$$

with $\nu$ the kinematic viscosity of water, equal to $1.141 \mathrm{e}-6 \mathrm{~m}^{2} / \mathrm{s}$ at $15{ }^{\circ} \mathrm{C}$. 
Such a high value of the Reynolds number indicates that the flow in this region is probably turbulent. Observations of the flow using the PIV technique confirm its turbulent feature, by the storage of instantaneous images. Figure 5 shows the velocity field near the catch. One can see birth, growth and movement of vortices caused by the catch.

In the vinicity of the netting however, one can compute the Reynolds number using the netting twine diameter as the reference length. It yields a Reynolds number of order $10^{2}$, which indicates rather a transitional flow in the wake of the twine elements.

Fig. 5. PIV Images of the instantaneous velocity field - open net - Zoom near the catch
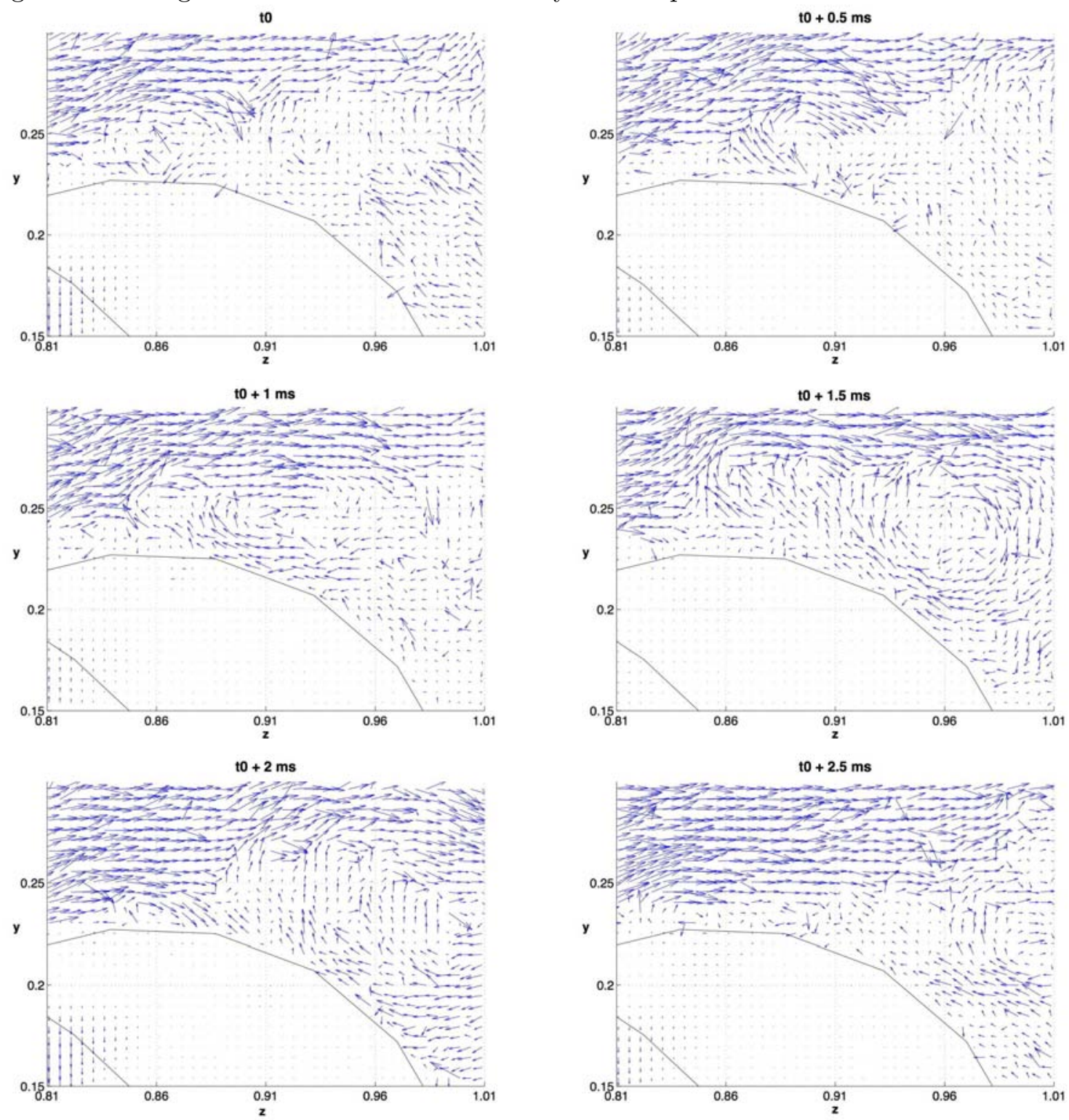

Then punctual LDV measurements were collected to reach more precise values of the turbulence rate in the different regions around the net. The LDV and PIV profiles will be presented in a second time. 


\subsection{LDV measurements}

LDV measurements allow to gather velocity data at different positions during a long time in order to access turbulent kinetic energy, which measures the velocity deviation to the mean.

Figure 4 shows the distribution of the measurement points around the geometry. The data acquisition time is 15 minutes per point to get significant means and standard deviations.

Recall that with the LDV technique available at Ifremer flume tank, the velocity components along the $z$ and $x$ direction are measured simultaneously (Fig. 2).

In case of points located on the symmetry axis, only the component along the $z$ axis is considered because of the axi-symmetric hypothesis of the net model and of the surrounding mean flow. The velocity components along the directions $x$ et $y$ are expected to be zero. As we shall see in the following table 1, this hypothesis is satisfied. For points 3, 4, 6, 7, 9 et 10, the $y$ velocity has to be measured. This has been carried out by placing the laser on the side of the model, in the plane $(\mathbf{O}, \mathbf{x}, \mathbf{z})$, with a strictly negative value of the $x$ laser location, equal to the $y$ component of the point, up to the sign. Then the velocity components measured are the ones along the $z$ and $x$ axes respectively, which correspond to the components along the $z$ and $y$ direction, thanks to the axi-symmetry assumption.

Fig. 6 displays the $z$ velocity components measured at points 1 and 12 . The $z$ velocity component at point 12 varies in an irregular pattern. It is a characteristic signature of turbulence. 
Fig. 6. LDV measurements for $u_{z}$ at point 1 and 12 during 50s

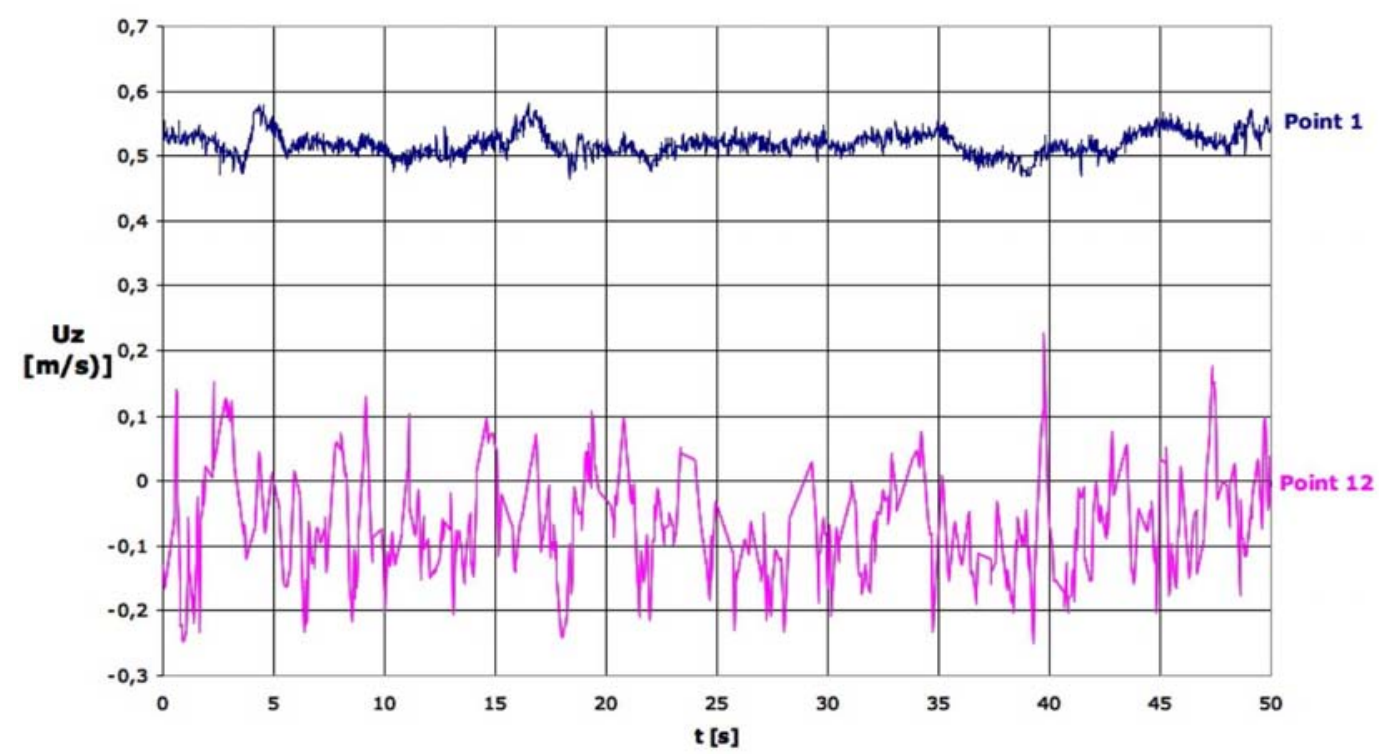


Tables 1 and 2 give the mean, the standard deviation and the turbulent kinetic energy at different locations.

\begin{tabular}{|c||c|c|c||c|c|c||c|}
\hline \multirow{2}{*}{\multicolumn{1}{|c||}{ Point }} & \multicolumn{3}{c||}{ Coordinates } & \multicolumn{2}{c||}{ Mean velocity components } & Mean velocity \\
\cline { 2 - 7 } & $\mathrm{x}$ & $\mathrm{y}$ & $\mathrm{z}$ & $\operatorname{mean}\left(u_{x}\right)$ & $\operatorname{mean}\left(u_{y}\right)$ & mean $\left(u_{z}\right)$ & 0.5122 \\
\hline 1 & 0 & 0 & -0.8 & -0.0025 & -0.0025 & 0.5122 & 0.4653 \\
\hline 2 & 0 & 0 & -0.012 & -0.0016 & -0.0016 & 0.4653 & 0.4891 \\
\hline 3 & 0 & 0.073 & 0.167 & -0.0024 & 0.0063 & 0.4891 & 0.4576 \\
\hline 4 & 0 & 0.043 & 0.167 & -0.0077 & 0.0196 & 0.4571 & 0.4941 \\
\hline 5 & 0 & 0 & 0.167 & 0.0047 & 0.0047 & 0.4940 & 0.4567 \\
\hline 6 & 0 & 0.104 & 0.404 & -0.0009 & 0.0251 & 0.4560 & 0.4551 \\
\hline 7 & 0 & 0.074 & 0.404 & -0.0030 & 0.0459 & 0.4528 & 0.4220 \\
\hline 8 & 0 & 0 & 0.404 & 0.0027 & 0.0027 & 0.4219 & 0.4281 \\
\hline 9 & 0 & 0.228 & 0.745 & -0.0159 & 0.2764 & 0.3266 & 0.2929 \\
\hline 10 & 0.011 & 0.178 & 0.745 & -0.0137 & 0.2078 & 0.2060 & 0.1121 \\
\hline 11 & 0.011 & 0 & 0.7 & 0.0086 & 0.0086 & 0.1114 & 0.0823 \\
\hline 12 & 0 & 0 & 1.036 & -0.0085 & -0.0085 & -0.0814 & 0.1895 \\
\hline 13 & 0 & 0 & 1.136 & 0.0015 & 0.0015 & -0.1895 & 0.0496 \\
\hline 14 & 0 & 0 & 1.336 & 0.0003 & 0.0003 & -0.0496 & 0.1752 \\
\hline 15 & 0 & 0 & 1.536 & -0.0074 & -0.0074 & 0.1749 & \\
\hline 1 & & & & & & & \\
\hline
\end{tabular}

Table 1

Mean velocity at the defined points.

Velocity vectors are drawn on figure 7 thanks to the data given in table 1 .

Fig. 7. Velocity vectors around the open net model

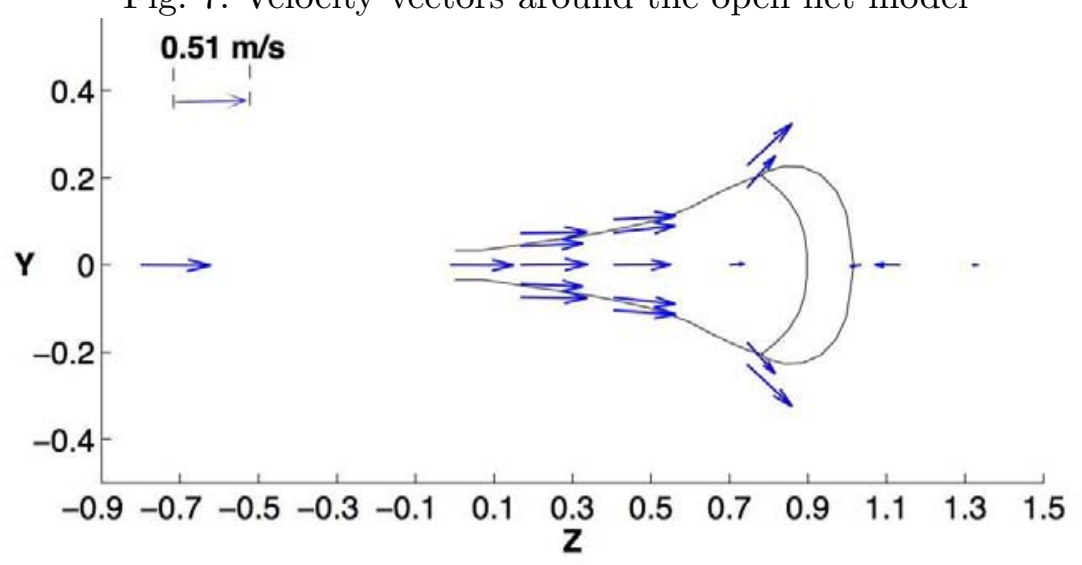




\begin{tabular}{|c|c|c|c|c|c|}
\hline \multirow{2}{*}{ Point } & \multicolumn{3}{|c|}{ Standard deviation } & \multirow{2}{*}{$\begin{array}{c}\text { TKE } \\
{\left[\mathrm{m}^{2} \cdot \mathrm{s}^{-2}\right]}\end{array}$} & \multirow{2}{*}{$\begin{array}{c}\text { Turbulence rate } \\
\qquad \%\end{array}$} \\
\hline & for $u_{x}$ & for $u_{y}$ & for $u_{z}$ & & \\
\hline 1 & 0.01489 & 0.01489 & 0.02543 & 0.00054 & 4.56 \\
\hline 2 & 0.01352 & 0.01352 & 0.02374 & 0.00046 & 4.21 \\
\hline 3 & 0.01614 & 0.01250 & 0.02404 & 0.00050 & 4.35 \\
\hline 4 & 0.03365 & 0.03286 & 0.05193 & 0.00245 & 9.67 \\
\hline 5 & 0.01097 & 0.01097 & 0.02628 & 0.00047 & 4.21 \\
\hline 6 & 0.01500 & 0.01286 & 0.02389 & 0.00048 & 4.28 \\
\hline 7 & 0.02172 & 0.02727 & 0.03060 & 0.00108 & 6.41 \\
\hline 8 & 0.02670 & 0.02670 & 0.0389 & 0.00147 & 7.49 \\
\hline 9 & 0.02120 & 0.02641 & 0.03553 & 0.00120 & 6.78 \\
\hline 10 & 0.03219 & 0.03685 & 0.03670 & 0.00187 & 8.44 \\
\hline 11 & 0.05895 & 0.05895 & 0.08725 & 0.00728 & 16.66 \\
\hline 12 & 0.110480 & 0.11048 & 0.08899 & 0.01617 & 24.82 \\
\hline 13 & 0.12860 & 0.12860 & 0.08957 & 0.02055 & 27.99 \\
\hline 14 & 0.17363 & 0.17363 & 0.11991 & 0.03734 & 37.73 \\
\hline 15 & 0.16960 & 0.16960 & 0.11194 & 0.03503 & 36.54 \\
\hline
\end{tabular}

Table 2

Turbulent kinetic energy.

\subsection{Analysis of the results}

First notice that the $x$ components are of the order of $10^{-3}$, which corresponds to the order of error committed by the experimentation, therefore satisfying the axisymmetric hypothesis previously set. Concerning points 10 and 11, notice a non zero value for the $x$ position for those points so as for the laser to avoid the twines.

The entrance velocity is given by the velocity at point 1 , that is $0.512 \mathrm{~m} / \mathrm{s}$. Inside the net, the flow is slowed down with the increase of $z$, mainly due to the proximity of the catch and also to the conical shape of the net. The velocity inside the net just before the catch (point 11 Tab. 1) is almost five times lower $(0.11 \mathrm{~m} / \mathrm{s})$ than the entrance velocity inside the net (point 5), which is equal to $0.49 \mathrm{~m} / \mathrm{s}$. It allows fish to easily hold their position in that region due to the reduced water flow, [8].

Concerning the points located inside a mesh, named 4, 7 and 10, the closer to the catch they are, the higher value the $y$ component has. It points out an increasing escapement of fluid through the meshes, linked to the increase in mesh opening and the proximity of the catch. This increase is in agreement with most of fish escape attempt locations, since they are preferably made through the wide-open meshes 
immediately ahead of the catch, [8].

Points 3,6 et 9, located in the $(\mathbf{O}, \mathbf{y}, \mathbf{z})$ plane, at a distance of $0.030 \mathrm{~m}$ above the net along the $y$ direction, suggest the bypass of the net by the fluid, as suggested by the increase in the $y$ velocity component (Tab. 1).

The natural turbulence in the tank is equal to $0.00054 \mathrm{~m}^{2} \cdot \mathrm{s}^{-2}$ (point 1 Tab. 2). One computes its root square, then divides it by the entrance velocity, $0.51 \mathrm{~m} / \mathrm{s}$ yielding $4.56 \%$. The higher turbulent rates are reached behind the catch, as expected $(\approx 38$ $\%)$. There is also a non negligible turbulent rate just in front of the catch, at point $11(16,66 \%$, Tab. 1), where the fluid is forced to bypass the catch and to flow out by the sides.

These punctual LDV measurements provide information on the turbulent kinetic energy but it is also interesting to access the influence of the net on the mean flow. To achieve this purpose, we collect LDV and PIV profiles at the different positions along the net.

\subsection{LDV and PIV profiles}

Velocity data were collected by the LDV technique along the six profiles drawn on figure 4 . The acquisition time was chosen equal to $100 \mathrm{~s}$ for each point, which seems a correct value to get a significant number of data. The shapes of the $z$ velocity profiles are given on figure 8.

Fig. 8. Shape of the component $u_{z}$ of the velocity along the profiles

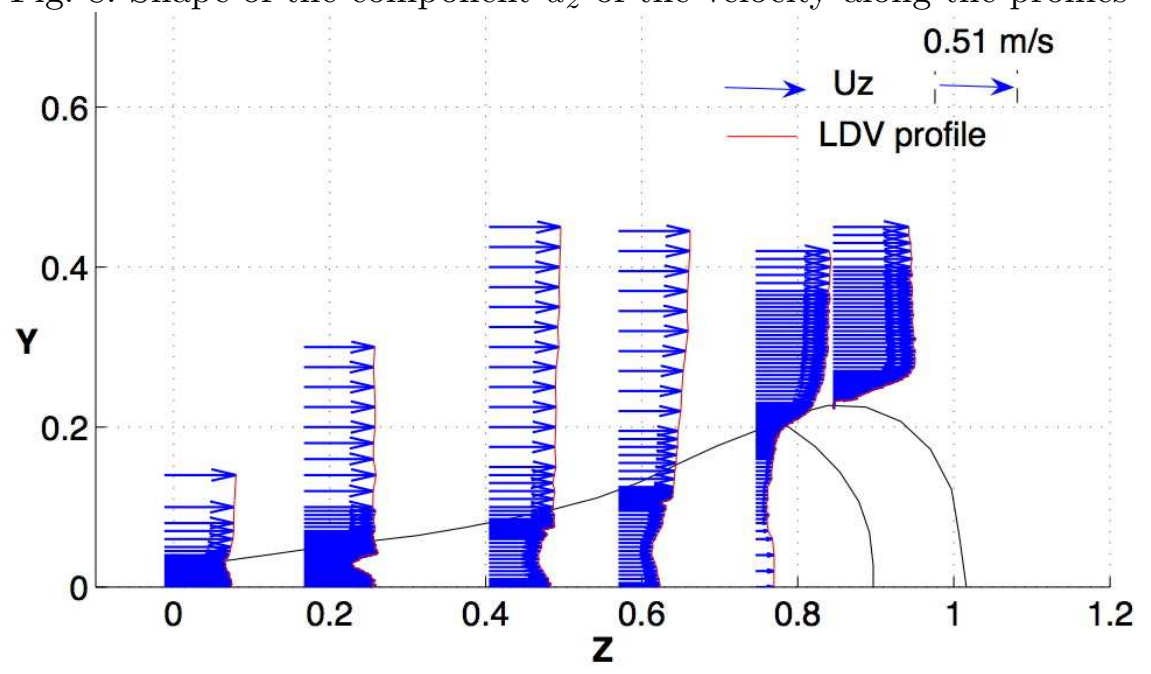

The same measurements were collected using the PIV technique. This technique is less accurate than the LDV. Nevertheless, the profiles obtained are close to those obtained with the LDV (Fig. 9). An advantage of the LDV technique is that it allows measurements inside the net, by passing the laser beam through the mesh. 
Fig. 9. LDV and PIV profiles - open net
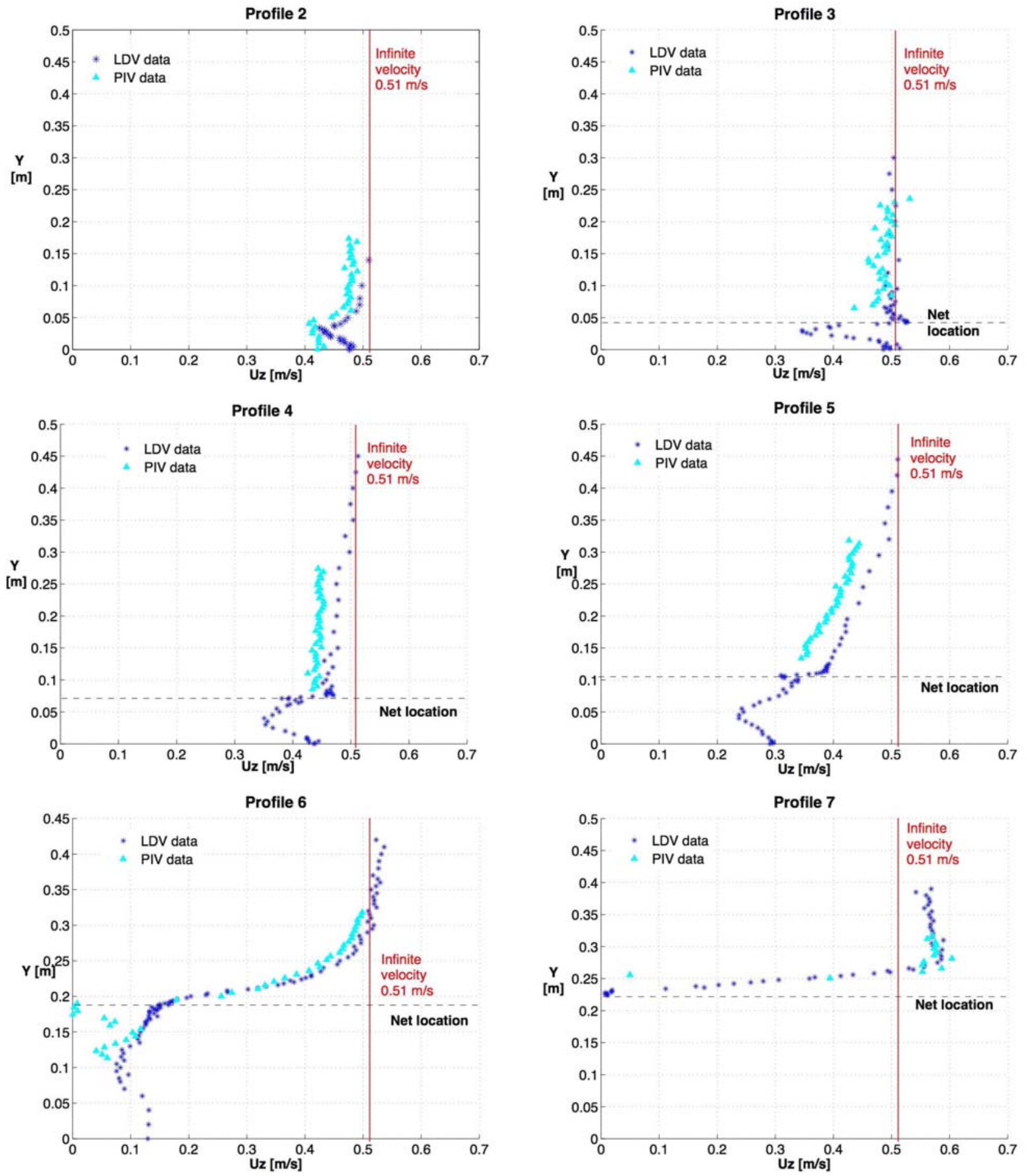

We observe on figure 9 a slowdown of the fluid at the net location, due to the frictions of the fluid on the twines and nodes. The conical shape of the net and the proximity of the catch also lead to a decrease of the internal net flow. On the contrary, the external flow is accelerated. It is enriched by the internal flow just before the catch, an impermeable obstacle that the fluid has to circle. 
Let us now consider what happens in the second configuration, when the net entrance is closed.

\section{Closed net model}

So as to achieve a better understanding of the effects of the outer net flow on the inner one, the entrance of the net was closed by a rigid cap-shaped foam (Fig. 3).

Temporal measurements were performed at some points, most of them at the same location as in the previous case (open net) and some new ones, like the points 16 , 17 et 18 (Fig. 4). Tables 3 and 4 display the value of the mean, standard deviation and turbulent kinetic energy for the different points. The velocity vectors given by table 3 are represented on figure 10 .

\begin{tabular}{|c||c|c|c||c|c|c||c|}
\hline \multirow{2}{*}{ Point } & \multicolumn{3}{c||}{ Coordinates } & \multicolumn{3}{c||}{ Mean velocity components } & Mean velocity \\
\cline { 2 - 7 } & $\mathrm{x}$ & $\mathrm{y}$ & $\mathrm{z}$ & $\operatorname{mean}\left(u_{x}\right)$ & $\operatorname{mean}\left(u_{y}\right)$ & $\operatorname{mean}\left(u_{z}\right)$ & 0.1028 \\
\hline 2 & 0 & 0 & -0.012 & -0.0214 & -0.02138 & 0.0983 & 0.4556 \\
\hline 4 & 0 & 0.043 & 0.167 & 0.0024 & -0.02377 & 0.4549 & 0.0981 \\
\hline 5 & 0 & 0 & 0.167 & 0.0256 & 0.02556 & -0.0912 & 0.4542 \\
\hline 7 & 0 & 0.074 & 0.404 & -0.00234 & 0.01610 & 0.4539 & 0.2181 \\
\hline 8 & 0 & 0 & 0.404 & 0.00345 & 0.0035 & 0.2181 & 0.3507 \\
\hline 16 & 0 & 0.220 & 0.570 & -0.00534 & 0.0949 & 0.3375 & 0.1158 \\
\hline 17 & 0 & 0 & 0.570 & $1.7 \mathrm{E}-05$ & $1.7 e-5$ & 0.1158 & 0.3044 \\
\hline 10 & 0.01075 & 0.1775 & 0.745 & -0.0177 & 0.2022 & 0.2269 & 0.0668 \\
\hline 11 & 0.01075 & 0 & 0.700 & -0.0172 & -0.0172 & -0.0622 & 0.2127 \\
\hline 18 & 0 & 0.170 & 1.196 & 0.0083 & 0.0083 & 0.2124 & \\
\hline
\end{tabular}

Table 3

Mean value at the different points - closed net

Fig. 10. Velocity vectors around the closed net model

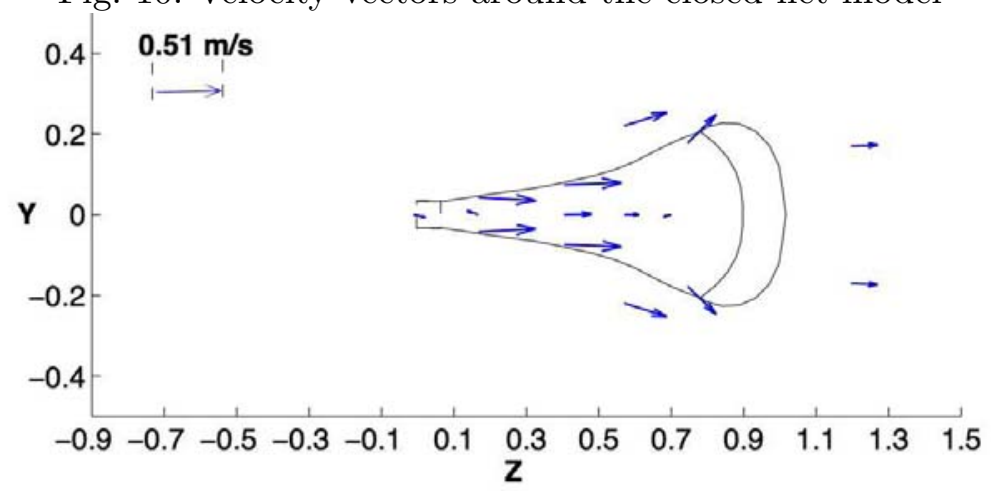




\begin{tabular}{|c|c|c|c|c|c|}
\hline \multirow{2}{*}{ Point } & \multicolumn{3}{|c|}{ Standard deviation } & \multirow{2}{*}{$\begin{array}{c}\text { TKE } \\
{\left[\mathrm{m}^{2} \cdot \mathrm{s}^{-2}\right]}\end{array}$} & \multirow{2}{*}{$\begin{array}{c}\text { Turbulence rate } \\
\%\end{array}$} \\
\hline & for $u_{x}$ & for $u_{y}$ & for $u_{z}$ & & \\
\hline 2 & 0.01384 & 0.01384 & 0.00643 & 0.00021 & 2.84 \\
\hline 4 & 0.04708 & 0.02711 & 0.05796 & 0.00316 & 10.97 \\
\hline 5 & 0.05970 & 0.05970 & 0.05150 & 0.00489 & 13.65 \\
\hline 7 & 0.02036 & 0.01821 & 0.02860 & 0.00078 & 5.46 \\
\hline 8 & 0.04707 & 0.04707 & 0.05149 & 0.00354 & 11.62 \\
\hline 16 & 0.02907 & 0.02970 & 0.05129 & 0.00218 & 9.11 \\
\hline 17 & 0.05758 & 0.05758 & 0.07328 & 0.00600 & 15.12 \\
\hline 10 & 0.04430 & 0.04925 & 0.05411 & 0.00366 & 11.81 \\
\hline 11 & 0.10118 & 0.10118 & 0.09739 & 0.01498 & 23.90 \\
\hline 18 & 0.15392 & 0.15392 & 0.09386 & 0.02810 & 32.73 \\
\hline
\end{tabular}

Table 4

Standard deviation and Turbulent kinetic energy.

As previously, LDV profiles were collected in this new configuration (Fig. 11). Fig. 12 displays the comparisons of the LDV profiles between the open net and the closed net configurations.

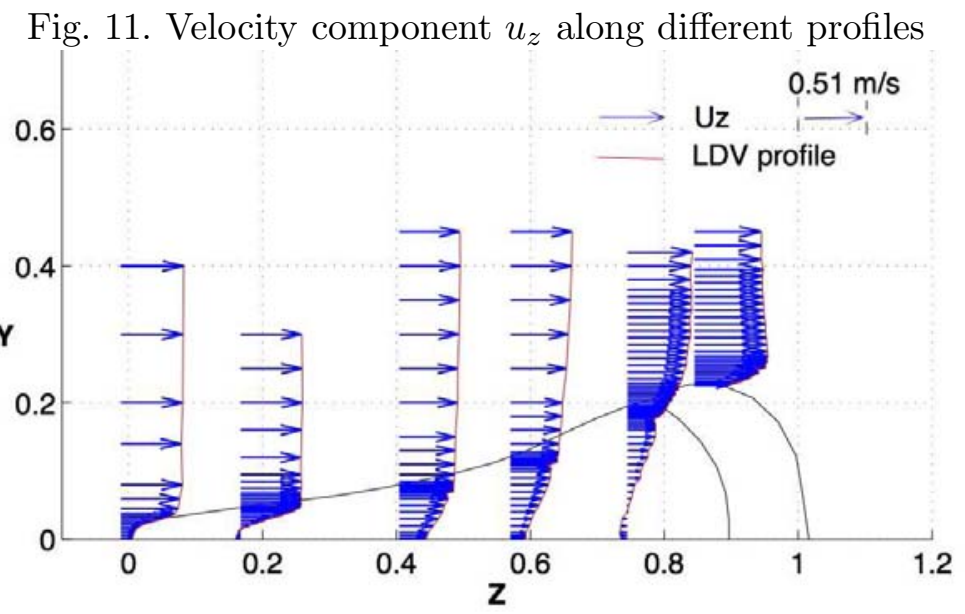

From these results, we can see that profile 2 corroborates the bypass of the closed entrance by the fluid. Notice (Fig. 12) that profiles 2, 3, 4 and 5 in the two configurations are surperimposed after a certain point inside the net. Between the net axis of symmetry and that point, the value of the $z$ velocity component is naturally lower in the case of the closed net than in the case of the open net. This means that the outer flow has an influence on the inner flow up to a point. Below that point, the flow is mainly fed by the fluid that comes by the entrance.

On profile 3 , the $z$ velocity component at the center (point 5 Tab. 3 ) is negative, which means that the fluid returns towards the entrance of the net. This is empha- 
Fig. 12. LDV profiles - closed vs open net
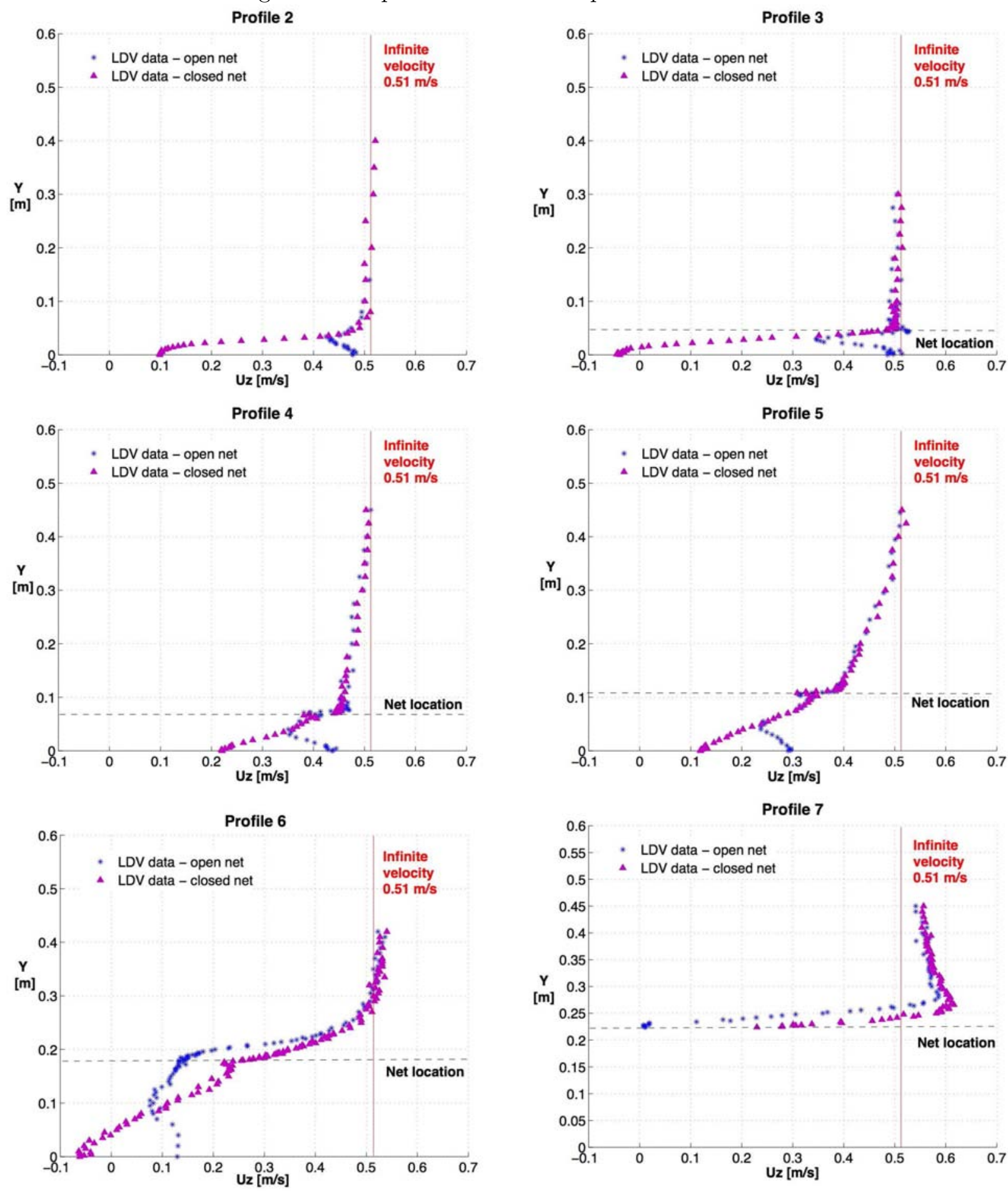

sized by a turbulent rate of $13.65 \%$ (vs $4.21 \%$ in the open net case). This is a local phenomena, since the $z$ velocity component is positive again at point 8 , equal to $0.21 \mathrm{~m} / \mathrm{s}$. Notice the $x$ and $y$ velocity components at points 5 and 11 are non negligible while they should be on account of the axi-symmetry assumption. This may be explained by a higher turbulence rate and lower value of the mean velocity for these points than with the open net. The negative value of the $y$ velocity component at 
point 4 , located on profile 3 , points out an entrance of fluid at this place, justifying the mixing at point 5 .

The $y$ velocity components are all the more important since the catch is closer $(0.2$ $\mathrm{m} / \mathrm{s}$ at point $10 \mathrm{vs} 0.016 \mathrm{~m} / \mathrm{s}$ at point 7$)$. The catch forces the fluid to leave through the open meshes.

Profile 6 displays a negative value of the $z$ velocity component at the center (point 11) of $-0.06 \mathrm{~m} / \mathrm{s}$. At that point, the turbulent rate is high: $23.90 \%$ (vs $16.66 \%$ in the open net case). It emphasizes a more important mixing due to the presence of the impermeable catch than it was the case with the open net. This is caused by a stronger influence of the outer flow on the inner one, due to the fact that this latter is not fed by the entrance. Because of this recirculating zone, more fluid escapes by the sides. It is confirmed by profile 7 , with the higher maximum value of the $z$ velocity component (more than $0.6 \mathrm{~m} / \mathrm{s}$ ) than for the case of the open net (below $0.6 \mathrm{~m} / \mathrm{s})$.

\section{Conclusion}

These experimental results confirm the turbulent feature of the flow in the vinicity of the catch and a more transitional one in the wake of the twines. They also show that the entrance of the cod-end net supplies most of the internal flow.

The fluid does not behave the same way when the net model is open or closed. In the case of the closed net, two recirculation zones are assumed inside the net. The first one is located closed to the entrance of the net. The fluid enters by the meshes, disordering the inner flow, yielding an increase of turbulence. This phenomena was not observed in the case of the open net, when the fluid tends to flow out of the net. The second recirculation is caused by the catch. The turbulence is higher at the net center just in front of the catch than it was with the open net. Then the velocity is lower and even negative. The fluid bypasses this turbulent zone, increasing the amount of fluid escaping through the meshes, therefore enriching the outer flow.

In both configurations, the zone of fluid escapement just ahead of the catch is also the one with the larger mesh opening, facilitating fish escapement by the sides, as confirmed by current knowledges on fish behaviour (see [8] for example or [16] based on observations at sea conducted during the PREMECS-II ${ }^{1}$ (PREdictive ModEl of Cod-end Selectivity) project). This is then a useful information to place selectivity mechanisms, like rigid grids, to allow juvenile fish to escape.

Schematically, the flow could be summed up by the streamlines drawn on figures 13 and 14 in both configurations.

$\overline{1 \text { http://www.ifremer.fr/premecs }}$ 
Fig. 13. Streamlines - open net

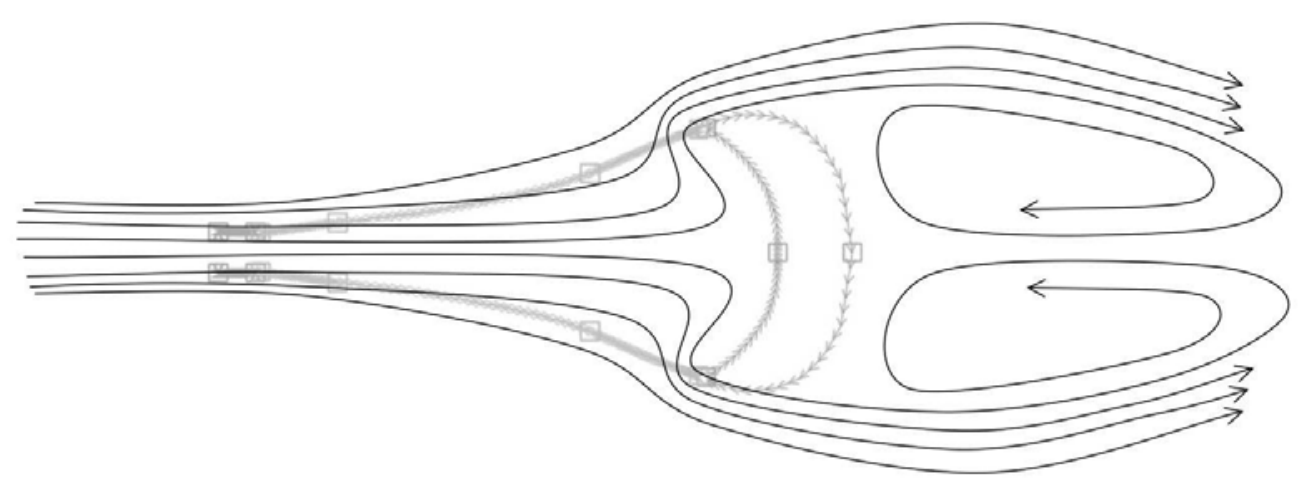

Fig. 14. Streamlines - closed net

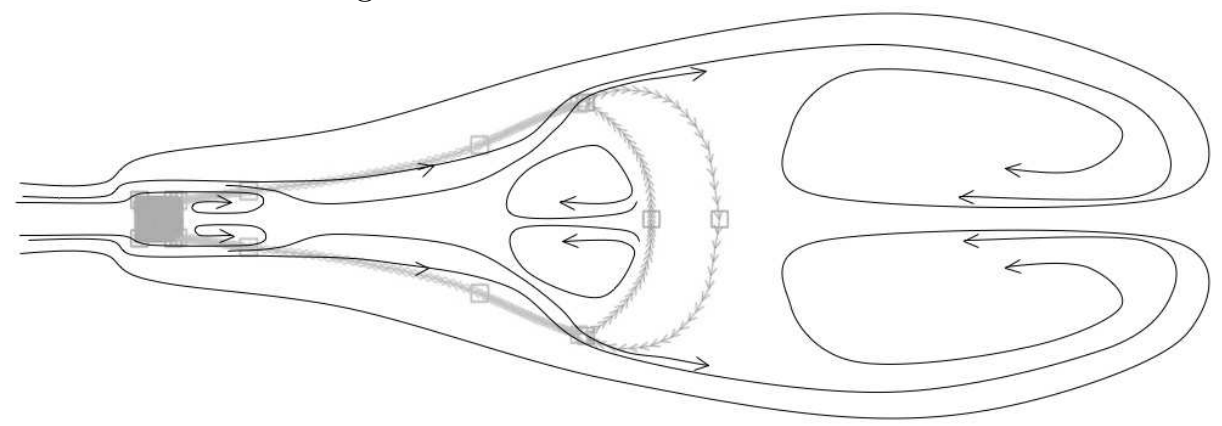

Such experimental data are currently used to validate models for flow around a codend net [10], [11]. They are very useful since they allow to fit the model parameters by comparison between experimental and numerical results.

Acknowledgements: We thank IFREMER and the region Bretagne for the financial support of this work. We are grateful to the Commission of the European Communities who has funded the project PREMECS II (Development of predictive model of cod-end selectivity, QLRT-2001-01328) which was really helpful to begin this study.

\section{References}

[1] G. -K. Batchelor, An introduction to fluid dynamics, Cambridge university press, 1973.

[2] N. Bahamon, F. Sardà, P. Suuronen, Improvement of trawl selectivity in the NW Mediterranean demersal fishery by using a $40 \mathrm{~mm}$ square mesh codend, Fisheries Research, Vol. 81, pp. 15-25, 2006.

[3] R. J. H. Beverton, Escape of fish through different parts of a codend, ICNAF Spec. Publ., No.5, pp 9-11.

[4] L. Botsford, J. Castilla, C. Peterson, The management of fisheries and marine ecosystems, Science, Vol. 277, 509-515, 1997.

[5] L. Coquelle, Simulation de comportements individuels instinctifs d'animaux 
dans leur environnement. De la description éthologique à l'exécution de comportements réactifs. Thèse ENIB, 2005.

[6] F. Durst, A. Melling, J. H. Whitelaw, Principles and Practice of Laser-Doppler Anemometry, Pergamon press, Oxford, 1976.

[7] G. Germain, J. V. Facq, D. Priour, Flow characterization around a cod-end, IMAM congress, Portugal, 2005.

[8] B. Herrmann, Effect of catch size and shape on the selectivity of diamond shape mesh cod-ends. I. model development. Fisheries Research, 71:1-13, 2005.

[9] B. Herrmann, Modelling and simulation of size selectivity in diamond mesh trawl cod-ends. PhD. Thesis. Aalborg University, Denmark, 2005.

[10] R. Lewandowski, G. Pichot, Numerical simulation of water flow around a rigid fishing net. Computer Methods in Applied Mechanics and Engineering, 196, pp 4737-4754, 2007.

[11] R. Lewandowski, G. Pichot, Sur l'écoulement turbulent au voisinage d'un filet de pêche. C. R. Acad. Sci. Paris, Ser. I. 344, pp. 141-145, 2007.

[12] R. Lewandowski, Analyse Mathématique et Océanographie, Masson, 1997.

[13] H. Le Dret, R. Lewandowski, D. Priour, F. Chagneau, Numerical Simulation of a Cod End Net. Part 1: Equilibrium in a Uniform Flow, Journal of Elasticity, Vol. 76, 139-162, 2004.

[14] F. G. O'Neill, S. J. McKay, J. N. Ward, A. Strickland, R. J. Kynoch, A. F. Zuur, An investigation of the relationship between sea state induced vessel motion and cod-end selection, Fisheries Research, 60, 107 - 130, 2003.

[15] F. G. O’Neill, L. H. Knudsen, D. A. Wileman, S. J. McKay, Cod-end drag as a function of catch size and towing speed. Fisheries Research, 72, 163 - 171, 2005.

[16] D. Priour, F. O'Neill, A. Sala, P. Chevallier, B. Hermann, PREMECS-II: Development of predictive model of cod-end selectivity, Final Report, Contract $n^{\circ}$ Q5RS-2002-01328, 2006.

[17] G. Pichot, Modélisation et analyse numérique du couplage filet-écoulement hydrodynamique en vue d'estimer la forme de la prise dans une poche chalut, $\mathrm{PhD}$ Thesis of Rennes 1 University, to be defended, 2007.

[18] D. Priour, Calculation of net shapes by the finite element method with triangular elements, Comm. in Num. Meth., Vol. 15, 755-763, 1999.

[19] F. Théret, Development of a predictive model of cod-end selectivity, Individual Progress Report IFREMER - Second Year - From December 97 to November 98, European project Fair Programme CT96 1555, 1998. 